Theatre Research in Canada

Recherches théâtrales au Canada

\title{
Ô Kanata
}

\section{Yves Sioui Durand et Catherine Joncas}

Volume 41, numéro 1, 2020

URI : https://id.erudit.org/iderudit/1071760ar

DOI : https://doi.org/10.3138/tric.41.1.149

Aller au sommaire du numéro

\section{Éditeur(s)}

Graduate Centre for the Study of Drama, University of Toronto

\section{ISSN}

1196-1198 (imprimé)

1913-9101 (numérique)

Découvrir la revue

\section{Citer ce document}

Durand, Y. S. \& Joncas, C. (2020). Ô Kanata. Theatre Research in Canada / Recherches théâtrales au Canada, 41(1), 149-151.

https://doi.org/10.3138/tric.41.1.149 d'utilisation que vous pouvez consulter en ligne.

https://apropos.erudit.org/fr/usagers/politique-dutilisation/ 


\section{Ô Kanata}

YVES SIOUI DURAND AND CATHERINE JONCAS

Les intentions de Robert Lepage et de madame Mnouchkine avec le spectacle théâtral Kanata pourraient paraître nobles. Nous sommes devant deux maîtres indéniables du théâtre.

Le théâtre du Soleil à travers ses créations, a souvent lutté pour un avancement des sociétés, des cultures, il a souvent plaidé pour le changement, pour la justice. Mais lorsqu'on se met au service d'une cause, on ne peut ignorer ceux-là même qui la portent.

Nous connaissons Robert Lepage personnellement pour avoir joué sur scène sous sa direction au CNA dans les années I990. Nous connaissons aussi madame Mnouchkine pour l'avoir rencontrée d'abord en 200 I au FTA et par la suite pour avoir eu le privilège d'assister au début des répétitions du Dernier Caravansérail à la Cartoucherie de Vincennes en 2004.

Nous sommes chez Ondinnok, parmi les fondateurs du théâtre autochtone au Canada. Nous avons été longtemps la seule compagnie de théâtre autochtone francophone de l'Amérique du Nord.

Pourquoi avoir choisi de nous ignorer? Nous sommes des artistes autochtones engagés. Nous avons une position, celle de la réappropriation de nos cultures et de nos formes d'expression en art et celle de la reconstruction culturelle de nos Nations.

En février 20I8, nous avons déposé un manifeste pour l'avancement des Arts et des artistes autochtones dans le cadre de la refonte de la politique culturelle du Québec. Nous y affirmions entre autre ceci : "La présence des arts et des artistes autochtones sur toutes les scènes et dans tous les théâtres et toutes les institutions de diffusion des Arts est la meilleure garantie contre l'ignorance, la discrimination et le racisme. "

Il fut un temps, après que l'armée américaine a définitivement écrasé les derniers autochtones libres, après l'extinction du bison d'Amérique et la mise en réserve, il fut un temps où le Wild West Show de Buffalo Bill tournait à travers l'Amérique du Nord et par la suite en Europe. Qu'est-ce qui attirait alors les foules? C'était le fait de pouvoir voir ces cruels et féroces Peaux Rouges, Sitting Bull et les autres, eux les authentiques résistants. Nous étions alors du spectacle, comme nous avons aussi habité les cages des expositions coloniales (ou zoo humains) du Bois de Vincennes.

Aujourd'hui, l'imposture de « jouer aux indiens » relève de l'insulte. Il existe au Canada une dramaturgie autochtone contemporaine, des créateurs et des acteurs de grande compétence.

Les temps ont changé et il doit y avoir réparation si on souhaite une véritable réconciliation.

Quel est votre pas vers la réparation?

La proposition du spectacle Kanata nous apparaît comme une aberration, une idée complètement déconnectée de la réalité et des enjeux du monde autochtone d'aujourd'hui au Canada. Vouloir raconter 450 années d'une histoire sanglante, tragique, en se glissant dans la peau d'un peuple qui en souffre encore, qui se bat et qui résiste à tous les jours et en parlant pour lui, est pour nous un non sens. 
Cette expression «se glisser dans la peau de l'autre » que vous employez pour qualifier le jeu du comédien qui pourrait représenter mieux que l'original, nous fait mal. Combien de bons prêtres, de bonnes sœurs, de sadiques de tout acabit, se sont glissés dans notre peau, manipulant nos corps et nos esprits, combien se sont glissés dans la peau et dans le corps de générations d'enfants des Premières Nations du Canada?

Les Peuples autochtones sont éveillés, mobilisés, déterminés à se réapproprier leur histoire, leur patrimoine esthétique et leurs codes!

Le temps où quiconque se met de longues tresses de laine noire et joue les indiens est révolu!

Les Premières Nations du Canada et leurs artistes n'ont pas besoin de votre compassion. Ils exigent justice et réparation. Ils demandent le respect. Catherine Joncas et moi, Yves Sioui Durand Huron-Wendat, nous avons cofondé Ondinnok, avec le Dehné John Blondin. Depuis trente-trois ans, nous nous battons pour le respect des protocoles, pour une reconnaissance de notre façon différente de concevoir l'art et pour la présence des autochtones sur scène.

Depuis le dépôt du rapport de la Commission vérité et réconciliation sur les pensionnats indiens en 2015, nous sentons une écoute de la part du gouvernement du Canada et un réel désir de changement au sein de la société dominante. Et tout à coup, le Théâtre du Soleil débarque chez nous et balaie du revers de la main tout ce lent et patient travail des artistes autochtones auprès des institutions, des musées, des théâtres et des gouvernements. La réconciliation est l'affaire de tous les gouvernements, de toutes les institutions, de tous les canadiens et d'autant plus celle des porteurs de visions de notre société.

Robert Lepage en est un représentant éminent et il ne peut ignorer ce devoir.

Le chanteur innu Florent Vollant, notre ami, nous a dit un jour : «Ils nous ont tout pris : nos territoires, nos enfants, nos animaux, notre avenir. Maintenant, ils veulent ce qui nous reste. Ils veulent nos larmes! »

La seule attitude qui nous semble permise à ce moment comme artistes autochtones dans ce débat autour de Kanata est l'acceptation de vos bonnes intentions, de votre génie doublée de gratitude.

Et après Kanata ... on tourne la page? L'histoire des autochtones aura été racontée et que les artistes, les comédiens, les metteurs en scène, les musiciens autochtones cessent de nous présenter leurs pièces, leurs œuvres si inabouties. Robert Lepage et Ariane Mnouchkine s'en sont occupés.

Aujourd'hui, vous venez rencontrer les artistes autochtones chez eux à Montréal, à Tiotiake, sur le territoire ancestral des peuples iroquoiens, le territoire des kanienkehakaronon, les gardiens de la porte de l'Est, qui y vivent toujours et dont nous reconnaissons la présence.

Je ne sais pas ce que vous attendez de cette rencontre.

Si c'est pour obtenir une collaboration, vous êtes deux ans trop tard. Si c'est pour reconnaître que peut-être bien un petit quelque chose vous a échappé, eh bien asseyez-vous et écoutez les nôtres ... Ouvrez votre esprit et votre cœur, le processus de décolonisation dans lequel nous sommes tous engagés ne s'arrêtera pas. Nous sommes le socle identitaire de ce pays et les trois Amériques sont toujours un continent amérindien. 
Si le théâtre comme vous dites Mme Mnouchkine est l'art de la présence, la présence de l'amérindien sur scène doit faire partie de cet Art et sa présence sur scène est un acte politique d'affirmation, de reconnaissance.

Y a-t-il quelqu'un d'éveillé parmi vous qui se demande pourquoi la plupart d'entre nous comme artistes autochtones avons choisi de ne pas parler maintenant des femmes disparues? La réponse? C'est par respect pour les familles qui souffrent alors que la commission d'enquête est toujours en cours et non pas par manque de talent ou d'imaginaire. Nous faisons partie de ces familles.

Comment d'aussi grands créateurs et artistes comme vous madame Mnouchkine et comme vous monsieur Robert Lepage ont-ils pu penser que «l'absence complète » d'acteurs autochtones du Canada dans votre production ne serait pas un enjeu majeur ici en terre d'Amérique? Pourquoi ne pas avoir songé à intégrer des artistes et des acteurs autochtones dès le début et à tous les niveaux, de la recherche à la production? (Ah oui, bien sûr, il y aura la présence de quelques-uns d'entre nous sur des vidéos et voilà cautionnée notre présence.)

La question au théâtre est de savoir qui parle? Qui parle dans Kanata?

On peut être solidaire d'une cause, mais on ne peut pas prendre la parole à la place de ceux qui portent cette cause. Par respect, on doit se mettre au service et c'est aussi ça le théâtre.

Bref, Kanata malgré les intentions de ses créateurs, reprend les façons de faire coloniales qui nous reléguaient aux rôles de figurants ou d'absents puisqu'à cette époque, c'était des imposteurs qui jouaient aux « indiens ».

Et cela, aujourd'hui au Canada est impossible, c'est une insulte à l'intelligence et à l'expression même de l'Art dans ce pays.

Niawenkowa!

\section{Yves Sioui Durand}

Co-fondateur d'Ondinnok, auteur, metteur en scène, réalisateur, artiste mentor

Prix du gouverneur général du Canada pour les Arts de la scène 2017

Compagnon des Arts et des Lettres du Québec 2018

\section{Catherine foncas}

Co-fondatrice d'Ondinnok, actrice, metteur en scène, artiste mentor

I6 juillet 2018 Cap aux Corbeaux 